\title{
¿Por qué no apreciamos nuestro patrimonio cultural arqueológico? El caso de Canto Chico, una comunidad de San Juan de Lurigancho en Lima, Perú
}

\author{
Why don't we appreciate our cultural heritage? \\ The case of Canto Chico, a community of San Juan de Lurigancho in Lima, Peru
}

\author{
Wilmer Mejía Carrión \\ Instituto Cultural Ruricancho, Lima 36 \\ E-mail: wilmermejiacarrion@gmail.com
}

Recibido el 16 de noviembre del 2016, aceptado el 12 de diciembre del 2016

DOI: https://doi.org/10.33017/RevECIPeru2016.0014/

\begin{abstract}
Resumen
A los peruanos siempre se nos dice "queramos lo nuestro", lo dicen los spots publicitarios de Prom Peru, lo mencionan siempre en los colegios en los diferentes niveles de instrucción escolar, se nos dice que Machu Picchu, Kuelap entre otros lugares, nos hacen especiales y por eso - como el resto de sitios arqueológicosdeben merecer nuestro respeto y cuidado pero parece que este llamado no tiene mayor repercusión, pues, a pesar de todo de este discurso, los peruanos seguimos maltratando el patrimonio cultural arqueológico. Así tenemos una diversidad de sitios arqueológicos diseminados a lo largo y ancho de nuestro país que están abandonados a pesar de encontrarse dentro de localidades plenamente habitadas. $Y$ más bien estas comunidades, lejos de convertirse en sus defensoras, se convirtieron en depredadoras del Patrimonio.

Para ejemplificar esta problemática, se eligió una localidad que es un buen ejemplo de esto. Canto Chico es una comunidad que se encuentra en el distrito de San Juan de Lurigancho, uno de los distritos más grandes de la ciudad de Lima. Esta comunidad creada en 1966, tiene una de los más emblemáticos sitios arqueológicos del distrito: La Huaca "Canto Chico" que data de la época inca (siglo XV d.C). Esta huaca, en un principio, era mucho más grande de lo que es ahora, el pueblo se asentó sobre ésta y así, con el pasar del tiempo desapareció gran parte de su infraestructura, (por ejemplo la comisaría y la iglesia de la comunidad se asientan sobre lo que fue la huaca) quedando, en la actualidad solamente una sección.

El Estado peruano - a través del Instituto Nacional de Cultura (ahora Ministerio de Cultura) trató de socializar el patrimonio arqueológico entre los vecinos de la comunidad con resultados infructuosos. Así la huaca ha estado en peligro desaparecer completamente en diversas ocasiones en manos de los propios vecinos. El artículo busca explicar por qué a pesar del proceso de socialización del patrimonio este no ha funcionado y el patrimonio arqueológico de esta localidad sigue siendo considerada un estorbo por sus miembros.
\end{abstract}

Descriptores: patrimonio cultural, Canto chico, San Juan de Lurigancho

\section{Abstract}

Peruvians always hear "we want what is ours", this is said by the publicity spots of Prom Peru, This always mention it in schools at different levels of schooling, the speech is the simple: Machu Picchu, Kuelap among other places, make us Special and therefore - like the rest of archaeological sites - should deserve our respect and care but it seems that this call has no greater repercussion, because, despite everything from this speech, 
Peruvians continue to mistreat the archaeological cultural heritage. Thus we have a diversity of archaeological sites scattered throughout our country that are abandoned despite being within fully populated localities. And rather, these communities, far from becoming their defenders, became predatory Heritage.

To exemplify this problem, a locality was chosen which is a good example of this. Canto Chico is a community located in the district of San Juan de Lurigancho, one of the largest districts of the city of Lima. This community created in 1966, has one of the most emblematic archaeological sites of the district: La Huaca "Canto Chico" dating from the Inca period ( $X V$ century $A D$ ). This huaca, at first, was much bigger than it is now, the people settled on this one and thus, with the passage of time disappeared much of its infrastructure, (for example the police station and the church of the community Settle on what was the huaca) being, at present only one section.

The Peruvian State - through the National Institute of Culture (now the Ministry of Culture) tried to socialize the archaeological heritage among the residents of the community with unsuccessful results. Thus the huaca has been in danger disappearing completely in several occasions in the hands of the own neighbors. The article seeks to explain why despite the process of socialization of heritage this has not worked and the archaeological heritage of this town is still considered a hindrance for its members.

\section{Keywords: cultural heritage, Canto Chico, San Juan de Lurigancho}

\section{Introducción}

El patrimonio cultural ha sido considerado por los diversos estados- nación como parte importante del desarrollo de una identidad colectiva nacional. Un caso emblemático en América Latina ha sido México, el cual luego de haber puesto en valor muchos sitios arqueológicos a lo largo de su territorio nacional diseñó instituciones que cumplieran con el propósito de difundir el conocimiento de este patrimonio entre su población.

El Perú no ha sido ajeno a este proceso y ha puesto en marcha diversos programas y proyectos de protección, conservación y difusión del patrimonio cultural. Ahora bien, parece ser que estos programas no han cumplido su propósito central pues existe una actitud ambigua e indiferente ( $y$ muchas veces destructiva) de los peruanos hacia su propio patrimonio.

Por un lado decimos sentirnos orgullosos de Machu Picchu (o de algún otro famoso monumento arqueológico), alabando su carácter de nueva maravilla del mundo y por otro denotamos una total indiferencia hacia muchas de estas manifestaciones culturales y es que el comportamiento destructivo y la actitud de descuido que tiene la población peruana hacia los monumentos arqueológicos contrastan completamente con la propaganda que lanzan las diversas entidades del rubro del turismo.

Todos los años desde la televisión y los diversos medios de comunicación vemos una serie de compactos spots publicitarios donde se nos dice "queramos lo nuestro". Sin embargo estos spots aunque muestran las bondades del país y su enorme potencial turístico llevan un mensaje que no encuentra mayor arraigo dentro de la población peruana: CUIDEMOS EL PATRIMONIO CULTURAL.

Estas actitudes en contra del patrimonio se hacen más evidentes en las localidades que contienen sitios arqueológicos. Las poblaciones aledañas a estos los ven como estorbos al desarrollo o como simples monumentos que muestran algo que estuvo allí, un pasado vago y mal conocido que, en la actualidad, no tiene nada que ver con ellos. Así tenemos un doble discurso, el oficial: Por un lado donde todos los peruanos debemos admirar el pasado, respetarlo y venerarlo porque es nuestro y es de todos. $Y$ por otro, lo real, los vestigios del pasado son maltratados y vejados tanto por la población como por las autoridades. $Y$ no es solamente cuestión de clases sociales, o niveles educativos, ya que R. Álvarez- Calderón nos muestra que La Huaca Pucllana en Miraflores tuvo que salvar varios obstáculos para ser lo que es ahora, uno de los más importantes atractivos turísticos del distrito de Miraflores. "En un plano de 1933...vemos que los planes originales de urbanización de la zona incluían un área destinada para una plaza o un parque, pero no la huaca Pucllana" [1] Así es que sea que se encuentren en zonas periféricas o de la Lima tradicional a pesar de ser huellas de un pasado milenario su deterioro, en la mayoría de casos, es inexorable.

En el ámbito político las autoridades se caracterizan por la indiferencia y descuido, o peor aún las mismas municipalidades atentan directa o indirectamente en contra de los monumentos arqueológicos reforzando la idea de su inutilidad para la sociedad, en los últimos 15 años por ejemplo se ha invertido en obras 
públicas, refacción de calles y demás obras de carácter material pero la realidad es que, salvo algunas excepciones los sitios arqueológicos siguen

tan abandonados como siempre.

¿Por qué? ¿Falta de conciencia? ¿Falta de educación?

Los peruanos tenemos un pasado milenario, no cabe duda, pero parece que no existe una idea clara de que hacer con él, ni siquiera el descubrimiento del Señor de Sipán y demás tumbas reales de la cultura mochica, ni el que se nos hable constantemente que nuestras culturas prehispánicas fueran tan espectaculares como la egipcia o la sumeria han transformado la forma de pensar de los peruanos. La desconexión entre el pasado y el presente está vigente en el Perú de comienzos del siglo XXI. El peruano no se siente relacionado con ese pasado ancestral y mágico que tanto hace alarde.

Es un tema complejo y por ello para entender el porqué de este comportamiento en la población, hemos tomado el caso del A.H Canto Chico en el distrito de San Juan de Lurigancho como muestra de lo que sucede en otras localidades del Perú, sobre todo los que se encuentran en el área periurbana, ya que en la actualidad esta zona contiene uno de los sitios arqueológicos más emblemáticos de ese distrito que a pesar de su importancia; para muchos de los vecinos de la zona y los Asentamientos Humanos que la circundan no representan más que un estorbo.

A partir de esta pesquisa buscamos apoyar nuestra hipótesis de trabajo: Las políticas culturales del Estado influyen en la valoración de los pobladores de Canto Chico hacia su patrimonio arqueológico.

\section{Metodología.}

Se elaboró una serie de historias de vida a partir de entrevistas a personajes clave dentro de la localidad como dirigentes $y$ vecinos principales que formaron parte de las primeras etapas de poblamiento de esta forma se obtendrá información sobre su concepción del patrimonio arqueológico y permitir observar si está ha cambiado a través del tiempo a partir de los diversos esfuerzos que han hecho diversas instituciones por su puesta en valor, además se realizará encuestas a los vecinos que vivan cercanos a la huaca. En el caso de Canto Chico, el colaborador clave es un docente de Historia del nivel secundario que creó el mini-museo de Canto Chico y que en la actualidad se encuentra inoperante por una serie de problemas. Como poblador de Canto Chico conoce a los ex dirigentes y dirigentes de la zona, además se encuentra en la preparación de un libro sobre la historia de su comunidad.

Las entrevistas fueron tanto formales y estructuradas así como informales y libres.

\section{Entendiendo qué es el patrimonio cultural y su utilidad}

En esta sección se expone algunos conceptos clave para entender lo qué es el patrimonio cultural.

\subsection{De bien cultural a patrimonio cultural}

Existen diferentes formas de conceptualizar el patrimonio cultural. Muchos de los conceptos que se manejan con respecto a éste crean confusiones pues lo definen como si fuera idéntico al de bien cultural. Nosotros tomaremos la siguiente definición:

"[El patrimonio cultural] Está compuesto por todos los bienes culturales que hemos heredado y que se crean en el presente, a los cuales la sociedad les otorga una importancia histórica, científica, simbólica o estética. Es el conjunto de bienes materiales e inmateriales, testigos o testimonios, vinculados a hechos, episodios, personajes, formas de vida, religión, trabajo, usos y costumbres que ilustran el pasado y que de una u otra forma aclaran o gratifican la identidad de una nación". [2]

Así pues, por un lado consideramos como bienes culturales a todos aquellos objetos que expresen la cultura de determinada sociedad- sea ésta del pasado o del presente-. Mientras que, consideramos como Patrimonio Cultural a todos aquellos bienes culturales que son reconocidos por los miembros de determinada sociedad como parte de su herencia colectiva otorgándole un determinado significado y valor simbólico. Podemos decir que, todo patrimonio cultural es un bien cultural pero no todo bien cultural es necesariamente patrimonio cultural [3].

Quedando en evidencia entonces que, para que un objeto pase de ser un bien cultural a ser patrimonio cultural, debe existir un proceso de valoración o patrimonialización y ésta debe provenir de la misma comunidad que lo alberga. Por tanto, si un grupo social no le confiere cierto valor simbólico a un objeto, por más antiguo que sea y por más legislado que esté, no sería un patrimonio cultural propiamente dicho puesto que: 
"El concepto de patrimonio cultural es subjetivo y dinámico, no depende de los objetos o bienes sino de los valores que la sociedad en general les atribuyen en cada momento de la historia y que determinan qué bienes son los que hay que proteger y conservar para la posteridad". (Las negritas son mías) [4]

Una vez que un grupo social le haya dado valor, éste entiende que el patrimonio cultural es algo que se hereda y que se dejará en herencia a las siguientes generaciones. Es decir, son los miembros de la localidad, los que deciden si un bien cultural merece ser patrimonio cultural, debemos ser claros en decir que el patrimonio cultural es una creación colectiva, si ese colectivo no conoce ni reconoce algún bien cultural como patrimonio cultural, no existe patrimonio cultural alguno.

\subsection{El Patrimonio cultural como construcción social: El proceso de patrimonialización}

Para que un objeto o monumento pase de ser un bien cultural a ser patrimonio cultural, tiene que existir un proceso de valoración al que llamaremos "proceso de patrimonialización". Me voy a centrar en éste, pues siguiendo a Ingrid Díaz Moreno:

"Al hablar de procesos de patrimonialización, antes que de patrimonio en sí mismo, busco dar cuenta precisamente del proceso a través del cual ciertos objetos, eventos, manifestaciones, prácticas cotidianas y elementos de la naturaleza son producidos e intervenidos como patrimonio" [5].

Aunque este proceso se da dentro de un pacto entre diferentes actores sociales -en nuestro caso entre los representantes del Estado- nación (Gestores sociales o culturales, y científicos sociales, entre otros) y los vecinos de la comunidad en la cual se encuentra algún bien cultural-, es este último- la comunidad- el actor social principal de este proceso ya que ésta debe conocerlo y reconocerlo como parte de su identidad. Es decir que es necesario que la comunidad conozca los diversos aspectos (históricos, artísticos entre otros) de un determinado bien cultural y se reconozca como heredero de éste ya que sin este conocimiento y reconocimiento de la población hacia un determinado bien cultural no puede haber proceso de patrimonialización alguno. Este bien, pues, tiene que tener algún sentido, algún significado para la comunidad que lo alberga.
"... la cultura está llena de sentido. Aunque la conducta puede ser automática y las sanciones dadas por supuestas, cualquier forma aceptada de acción o de creencia, cualquier institución dentro de una cultura "tiene sentido". (...)La experiencia se define culturalmente, definición que implica que la cultura tiene un significado para los que viven de acuerdo con ella. Hasta para los bienes materiales la definición y captación de su función llega a entrar culturalmente en la vida. Un objeto, tal como una mesa, figura en la vida de un pueblo únicamente si es reconocido como tal. Para un miembro de una aislada tribu de Nueva Guinea serían tan incomprensibles como el simbolismo de sus dibujos lo sería para nosotros. Sólo después que un objeto ha cobrado sentido mediante explicación, definición y captación de su función llega a entrar culturalmente en la vida. "[6]

Entonces el que un objeto pase a tener sentido o significado - o en nuestro caso, el hecho de que un bien cultural se convierta en patrimonio cultural-, lejos de ser obra de una imposición legal, es un proceso vivo entre diversos actores sociales, una obra colectiva, producida por el conjunto de la sociedad.

"Hay que convenir que el patrimonio, como resultado de una construcción social, refiere un conjunto de bienes culturales que reciben una valoración positiva por parte de la sociedad, cuya identidad expresan, en el sentido que es un elemento mediante el que se establece la diferencia con los otros grupos sociales y culturales (...) El patrimonio, una especie de simbología social para el mantenimiento y la transmisión de la memoria colectiva, está constituido por los bienes representativos de cada sociedad. $Y$ se puede afirmar que los fenómenos patrimoniales revisten una triple dimensión: física, social y mental; es decir, material y simbólica". [7]

\subsection{Valor Material y valor simbólico}

Si bien es positivo que los arqueólogos deseen trabajar con la comunidad por su misma formación profesional, los arqueólogos se verían limitados en el desarrollo de proyectos de la llamada "arqueología pública" la antropología tendría un rol más decisivo en este tema lo que ha sido soslayado en los escritos de la mayoría de los arqueólogos. Son los antropólogos - por su propia formación profesional en trabajos de convivencia y entendimiento de la cultura y dinámicas sociales de una comunidad- los que estarían mejor capacitados para la formación de este tipo de proyectos. 
Pues bien, podemos ver que, por un lado, que el bien cultural monumental vendría ser una manifestación de la cultura de nuestros antepasados, son vestigios de una época pasada gracias a los cuales podemos comprender y entender la cultura que proviene de ese pasado. Es decir gracias a los bienes culturales podemos aproximarnos hacia sus pensamientos, creencias, sentimientos. Ahora bien para que este bien, se convierta en patrimonio - tal como los hemos expuesto líneas arriba- debe ser parte de la cultura y las dinámicas sociales de la localidad del presente.

En principio, a diferencia de los arqueólogos, los antropólogos no le dan tanto énfasis a la parte material del patrimonio cultural sino al lado simbólico de éste. Es el valor simbólico que emana del objeto lo que es importante para los antropólogos y es a partir de éste que se trabaja.

"El hecho de hablar de patrimonio cultural y no de patrimonio se relaciona con las aportaciones de la antropología y con la evolución de los conceptos de cultura y de identidad (...) Desde la antropología se considera que el patrimonio está formado por objetos que perduran en el tiempo. Y como que el paso del tiempo es la sustancia de la historia, hay que concebir el patrimonio como a conjunto de objetos de la historia. Estos objetos son los vehículos de una serie de significados, tienen una carga simbólica que se adopta según la percepción de los receptores, los encargados de patrimonializarlos $y$, por lo tanto, de convertirlos en heredables. Pero no se debe olvidar que la relación y la vinculación que se establece entre los elementos patrimoniales y las comunidades (sociedades, grupos, etc.) son una construcción social y, en consecuencia, "todo el mundo es libre de aceptar o de rechazar, toda o en parte, la herencia. Por lo tanto, no es suficiente con transmitir, es necesario que quien lo hereda lo acepté. Debe haber la voluntad de querer heredar. Cuando los elementos patrimoniales se asocian con una identidad y con unos valores e ideas, entonces se sacralizan y adquieren un carácter casi inmutable. Es decir, se convierten en un símbolo a partir del que se puede expresar de una manera sintética $y$ efectiva la relación entre las ideas, los valores, las concepciones, las creencias, y se puede convertir todo en emociones. Así, la selección de los elementos patrimoniales es producto de los discursos identitarios existentes; por lo tanto, no es neutral ni objetiva sino que está cargado de valores. Intentar establecer el retrato del tratamiento educativo del patrimonio cultural en un momento determinado puede ser una empresa difícil, ya que el concepto de patrimonio cultural "está experimentando continuamente un proceso de deconstrucción y de construcción, seguramente porque no es un medio o un recurso para conocer el pasado o para hacer historia, sino que el patrimonio es el pasado evidenciado, es la pervivencia y aquello que queda visible del pasado, y que desde el presente se le otorga valor.

Pienso que para la didáctica de las ciencias sociales, el patrimonio puede considerarse la expresión más genuina de la identidad, porque su apropiación por parte de las personas puede favorecer: - la construcción de una identidad ciudadana responsable (personal, social y cultural) fundamentada en la voluntad de respeto $y$ de conservación del entorno y del pasado; - el desarrollo de un pensamiento social crítico, para ser capaz de situar históricamente las evidencias del pasado y darles significado social, político y cultural; - la capacidad de implicarse y de actuar de manera responsable en la conservación, la preservación y la divulgación del medio local y global; y - la construcción de un conocimiento histórico y social, a partir del establecimiento de la continuidad temporal (pasado-presente-futuro), de la construcción de la conciencia histórica y de la indagación histórica con fuentes primarias. [8]

Entendida de esa manera es posible comprender entonces que lejos de ser algo muerto es parte de la cultura actual y la dinámica social de la localidad, ya que va a tener una función social dentro de ella, y por tanto valor:

"Se podría afirmar que patrimonio puede ser cualquiera objeto, pero no hay que no olvidar que el concepto incluye una elección, una selección. Así, por poder atribuir a un objeto la categoría de patrimonio, es necesario que reúna una serie de valores que se pueden resumir en tres (el valor de uso, el valor formal y el valor simbólico): - el valor de uso se refiere a la utilidad que tiene el objeto, es necesario que cubra algún tipo de necesidad; - la formal, hace referencia a la atracción que debe despertar a los sentidos, tanto por la forma, el volumen, etc.; $y$ - el simbólico es determinante por el hecho de ser testigo de ideas, de hechos o de situaciones del pasado y que permite conectar a personas de tiempo diferentes" (...)

Pero ¿Qué criterios tendríamos que establecer para saber que es patrimonio y que no? (...) nos dice: 
"También desde la antropología se han hecho propuestas para establecer los criterios que permiten a los elementos culturales convertirse en patrimonio. Existen básicamente dos tendencias. Una que opta por la pérdida de la funcionalidad (la obsolescencia), la escasez y la nobleza. Pero parece que el consenso se encuentra en una propuesta basada en la naturaleza, la historia y la inspiración creativa o genialidad. Se piensa que estos criterios pueden ser extraculturales y universales, porque funcionan siempre para determinar si un elemento es patrimonializable o no. Estos criterios consisten en (Prat, 1993: 22-29): - la naturaleza entendida como la referencia a la existencia de unos poderes que no dependen del orden social y que escapan del control humano; - la historia, el pasado, que escapa del control humano, se mitifica y hace que nos sentamos unidos por una dependencia unidireccional de filiación que convierte sus testigos en las reliquias del presente; $y$ - la inspiración creativa o la genialidad que representa la excepcionalidad cultural, la individualidad de un personaje que rompe con las reglas y las capacidades culturales del gran colectivo, hecho que permite reafirmar la fuerza del individuo por encima del grupo. Cuando estos criterios confluyen en los elementos culturales (tanto materiales como inmateriales) y se asocian con una identidad $y$ unas determinadas ideas $y$ valores, hacen que esta identidad, estas ideas, estos valores, se sacralicen y adquieran un carácter casi inmutable. En definitiva, la principal virtualidad es la de convertirse en un símbolo porque expresa de manera sintética y efectiva la relación que debe haber entre él y las ideas y los valores cuando las concepciones y las creencias se transforman en emociones. Estos tres criterios configuran un triángulo que da valor patrimonial a los objetos”. (...)

También podemos hablar de un proceso de patrimonialización, ya que para que este sea considerado como patrimonio en sí, es necesario que la localidad lo vea como tal, porque al final de cuentas si la sociedad no se apropia de este no es patrimonio.

\section{Resultados y discusión}

A partir del marco teórico expuesto es claro que no se ha llevado a cabo un completo proceso de patrimonialización por parte del Estado de los bienes culturales arqueológicos de la comunidad de Canto Chico. Los agentes del Estado peruano han actuado de manera ambigua, por un lado permitiendo que el sitio arqueológico sea destruido y luego tratando de conservar lo poco que queda de él.
En ninguna de las dos ocasiones ha habido un esfuerzo por entender a profundidad las necesidades locales y a partir de ésta lograr insertar el bien cultural en la dinámica cultural de la localidad de Canto Chico.

La única que vez que se entendió la relación entre las necesidades de la población y el bien cultural en proceso de patrimonialización se dijo a los vecinos que si no cuidaban lo poco que quedaba del sitio arqueológico no les darían los títulos de propiedad. Una vez que obtuvieron sus títulos de propiedad, el sitio arqueológico - aunque ya tenía un cerco perimétrico- dejo de ser resguardada y los afanes por destruirla y convertirla en algo más "productivo" - como un mini estadio- continuaron.

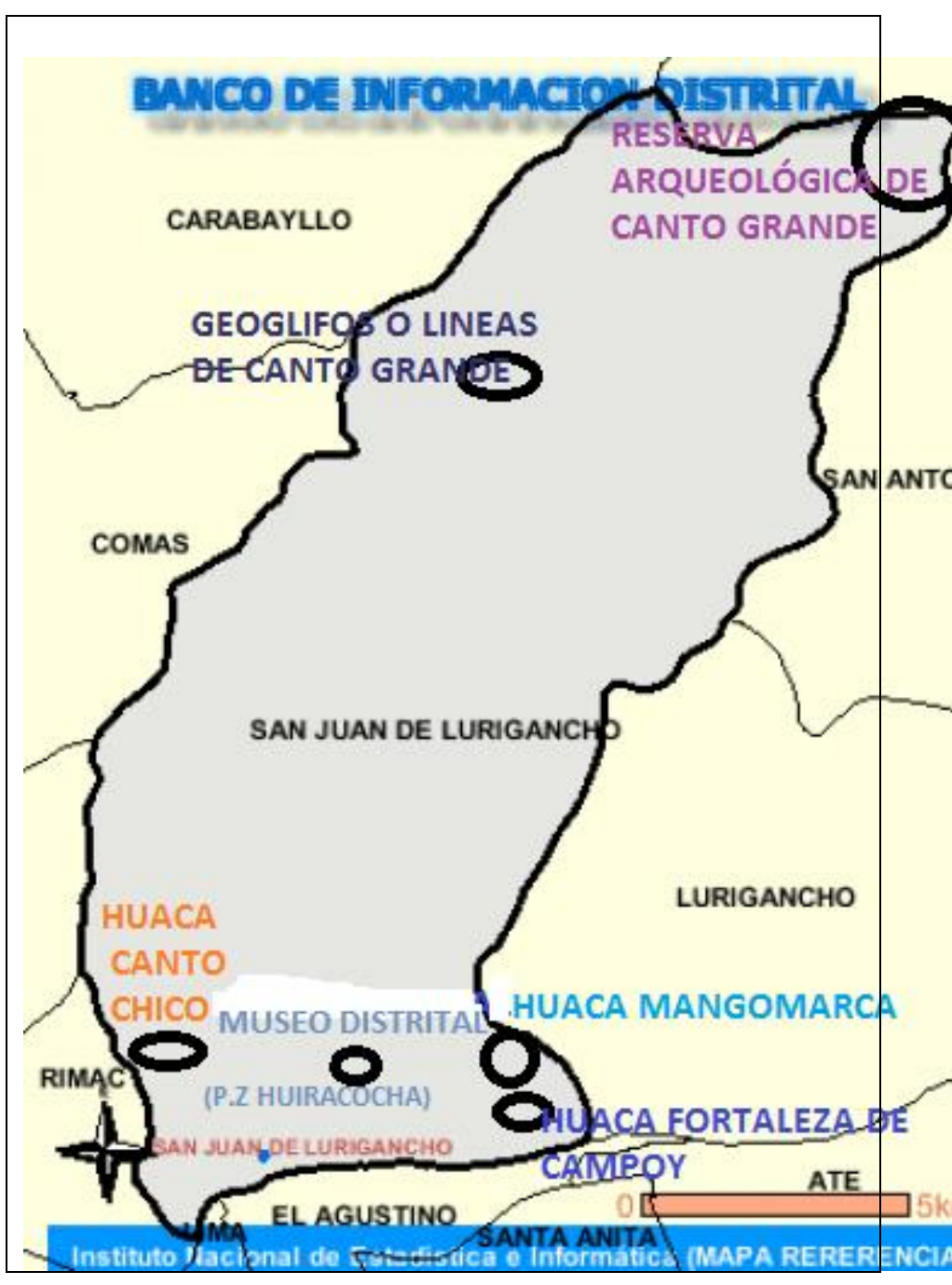

Figura 1: Mapa de San Juan de Lurigancho que muestra donde se encuentran los diversos sitios arqueológicos del distrito. Resaltado en anaranjado; Huaca Canto Chico.

Elaboración propia sobre mapa del Instituto Nacional de Estadística e Informática 


\section{Resultados y discusión}

A partir del marco teórico expuesto es claro que no se ha llevado a cabo un completo proceso de patrimonialización por parte del Estado de los bienes culturales arqueológicos de la comunidad de Canto Chico. Los agentes del Estado peruano han actuado de manera ambigua, por un lado permitiendo que el sitio arqueológico sea destruido y luego tratando de conservar lo poco que queda de él.

En ninguna de las dos ocasiones ha habido un esfuerzo por entender a profundidad las necesidades locales y a partir de ésta lograr insertar el bien cultural en la dinámica cultural de la localidad de Canto Chico.

La única que vez que se entendió la relación entre las necesidades de la población y el bien cultural en proceso de patrimonialización se dijo a los vecinos que si no cuidaban lo poco que quedaba del sitio arqueológico no les darían los títulos de propiedad. Una vez que obtuvieron sus títulos de propiedad, el sitio arqueológico - aunque ya tenía un cerco perimétrico- dejo de ser resguardada y los afanes por destruirla y convertirla en algo más "productivo" - como un mini estadio- continuaron.

Ejemplo de esto, es que en una de las entrevistas se mencionó que: "vino una vez un candidato a la alcaldía y prometió construir sobre este sitio en un mini estadio... inclusive algunos adelantándose a este hecho por las noches empezaron a picar, destruyendo parte de la huaca".

Es así como las políticas culturales del Estado se han enfocado demasiado en salvaguardar el patrimonio cultural arqueológico sin tomar en cuenta las necesidades de la población de Canto Chico, y en los casos que lo han hecho ha sido tomando una necesidad de corto plazo como es el tener un título de propiedad, una vez que esta necesidad quedó totalmente satisfecha terminó la utilidad del bien cultural para la comunidad desapareciendo la voluntad de heredarlo.

Esto nos habla de un proceso de patrimonialización mal conducido por parte de los agentes del Estado que no han querido conocer ni relacionarse con la comunidad que alberga los bienes culturales.

Los habitantes de Canto Chico no son descendientes de los pueblos que construyeron los bienes culturales que ahora alberga sin embargo existe una relación entre la población migrante de origen andino y amazónico y la población prehispánica del valle de Lurigancho, ambos tienen una raíz originaria común compartiendo -a pesar del tiempo y las diferencias regionales- ciertos elementos culturales similares (gastronomía, medicina tradicional, etc.) sobrevivientes al periodo colonial y republicano. Encontrar un puente, es decir una relación entre ambos es fundamental para que nos veamos parte de un mismo proceso.

Además los habitantes actuales de Canto Chico pueden aprender muchas cosas de cómo vivían las poblaciones prehispánicas locales y así entender que éstas lejos de ser "atrasadas" como da a entender un discurso de poder tenían un dominio de su territorio y de su medio ambiente que les permitía aprovechar al máximo su medio ambiente, convirtiendo parte de la desértica quebrada de Canto Grande en un valle artificial que sobrevivió hasta la década de los 70's.

La función social del patrimonio cultural debe enfocarse no solamente en necesidades materiales sino también a necesidades en cuanto a la conformación de identidad, ciudadanía, conciudadanía y autoestima social.

\section{Conclusiones}

El entender que el patrimonio cultural es una construcción social, nos permite tener un buen punto de partida, para preparar el proceso de patrimonialización (o socialización patrimonial) pues nos ayuda a comprender que si la población aledaña no ve ningún significado a los bienes culturales prehispánicos que tiene en su localidad no se debe necesariamente a que tenga un bajo nivel educativo sino a que no conoce su valor simbólico e histórico y por tanto no se reconoce en él.

Para que los bienes culturales pasen a convertirse en patrimonio cultural, se debe tener en cuenta las dinámicas sociales y culturales de la localidad, sus necesidades a partir de las cuales se puede insertar los bienes culturales dentro de la dinámica social y cultural y así cumplir una función social dentro de la localidad.

\section{Agradecimientos}

Agradecimiento a mis padres que gracias a su infinita comprensión y apoyo me permiten seguir adelante. 


\section{Referencias}

[1] R. Álvarez-Calderón, De cómo fue casi demolida la Huaca Pucllana en ARKEOS Revista Electrónica de Arqueología de la PUCP http://mileto.pucp.edu.pe/arkeos/content/view/2 33/26.. (consultada el 2 de febrero de 2013)

[2] O. Fontal (coord) La educación patrimonial. Teoría y práctica en el aula, el museo e Internet, Gijón, Ediciones Trea SL, (2003)

[3] Este concepto muestra lo que deseo afirmar: bien cultural y patrimonio cultural son conceptos diferentes, donde el de patrimonio incluye al de bien, es decir el concepto de patrimonio es más amplio. "El Patrimonio Cultural de Aragón está formado por Bienes Culturales que representan nuestra identidad histórica, artística, cultural y natural. Estos Bienes conforman los elementos que los aragoneses identifican con su pasado, su presente y el legado que deben transmitir a las generaciones futuras como herencia de su cultura"(Las negritas vienen de origen) Fuente: http://www.patrimonioculturaldearagon.es/biene s-culturales (consultada el 9 de junio de 2012)

[4] Fuente: Página web del Instituto Andaluz del Patrimonio Histórico http://www.iaph.es/web/canales/patrimoniocultural/ (consultada el 15 de agosto de 2012)

[5] I. Díaz, Patrimonialización, construcción de Identidades y formación del Estado en Puerto Santander Y San Martín, Meta (Trabajo de grado presentado como requisito parcial para optar al título de antropóloga, Pontificia Universidad Javeriana Facultad de Ciencias Sociales Departamento de Antropología Bogotá (2010)

[6] M. Herskovits, El hombre y sus obras, Fondo De Cultura Económica, México D.F (1956), p. 39; las negritas son mías

[7] J. Arévalo, El patrimonio como representación colectiva. La intangibilidad de los bienes culturales ( 2010), (las negritas son mías) http://www.ugr.es/ pwlac/G26_19Javier_Marco s_Arevalo.html. Consultado el 19 de julio de 2014

[8] N. González, El valor educativo y el uso didáctico del patrimonio cultural, Universitat Autònoma de Barcelona,http://pagines.uab.cat/neus.gonzalez /sites/pagines.uab.cat.neus.gonzalez/files/praxi s_neusgonzalez.pdf (consultada el 9 de junio de 2012) 